\begin{abstract}
Aim of the study: The aim of this study was to present our own experiences concerning risk factors for cardiac side effects in the study group.

Material and methods: The study was performed in 120 patients with HER2 overexpressing breast cancer who received immunotherapy in the Clinical and Experimental Oncology Department, between 2006 and 2011.

Results: LVEF reduction $>10 \%$ of the baseline fraction was observed in $10(8 \%)$ patients. Symptomatic heart failure occurred in two individuals. Due to persistent cardiotoxicity five patients (4\%) had to discontinue therapy prematurely. Risk factors for cardiac toxicity in the analyzed group included: previous radiotherapy to the left side of the chest $(p=0.05)$, higher BMI $(p=0.05)$, negative steroid receptor status $(p=0.045)$ and low baseline LVEF $(p<0.001)$. Patients receiving radiotherapy were more likely to develop cardiotoxicity if presenting older age $(p=0.0003)$.

Conclusions: Previous radiotherapy to the left side of the chest, negative steroid receptor status, high BMI and low baseline LVEF were associated with increased risk of cardiac dysfunction. There was no difference between patients receiving adjuvant therapy and those treated due to metastatic disease.
\end{abstract}

Key words: trastuzumab, cardiotoxicity, risk factors, breast cancer.

\section{Cardiac side effects of trastuzumab in breast cancer patients - single centere experiences}

\author{
Joanna Huszno, Dominika Leś, Danuta Sarzyczny-Słota, Elżbieta Nowara
}

Clinical and Experimental Oncology Department, Maria Skłodowska-Curie Memorial Cancer Center and Institute of Oncology, Gliwice Branch, Poland

\section{Introduction}

Trastuzumab is a humanized monoclonal antibody, selectively targeted at the extracellular domain of human epidermal growth factor receptor 2 (HER-2). Overexpression of HER2 or amplification of the HER2 gene occurs in about $20 \%$ of breast cancer patients [1]. Due to the widespread use of trastuzumab in breast cancer therapy, special caution in the qualification for treatment is necessary and also careful monitoring during patients' treatment to detect potential complications. One of the most common side effects of trastuzumab treatment is cardiotoxicity manifested as heart failure, accompanied by a decrease in left ventricular ejection fraction (LVEF) or an asymptomatic decrease in LVEF. The incidence of myocardial dysfunction during trastuzumab monotherapy is estimated at about 4-7\%, including about 3\% for toxicity grade 3 and 4 according to CTCAE (Common Terminology Criteria for Adverse Events) [2, 3]. Cardiotoxicity in treatment regimens containing anthracyclines can even reach $27 \%$ [3]. As opposed to the risk of cardiac side effects developing as a consequence of anthracycline treatment, the risk of trastuzumab-induced cardiomyopathy does not depend on the total dose of the drug [4]. The mechanism underlying trastuzumab-induced cardiomyopathy is not entirely clear. Some authors believe that it may be linked to the presence of the HER2 receptor on the surface of cardiomyocytes. Human epidermal growth factor receptor 2 is a receptor which plays an important role in cardiogenesis and myocardial protection against harmful factors such as ischaemia or adrenergic stimulation. Trastuzumab blocks the HER2 receptor by binding to its extracellular domain. Suppression of signal transduction inhibits repair mechanisms and promotes cardiomyocyte apoptosis [5]. Prior use of anthracyclines reduces the number of receptors on the surface of myocardial cells, which then are additionally blocked by trastuzumab [6]. Echocardiography is a routine method for monitoring cardiac side effects. Assessment includes measurement of parameters for systolic and diastolic function as well as anatomical cardiac dimensions. Simpson's method is recommended for assessment of ejection fraction [7-9]. Abnormalities of right ventricular contractility, ventricular dilation and abnormalities of left ventricular contractility are the earliest manifestations of myocardial damage diagnosed by echocardiography. In case of clinical symptoms of heart failure, therapy must be stopped as well. Patients with an asymptomatic decrease in LVEF by $15 \%$ (or by $10 \%$, if the acceptable lower LVEF limit is assumed as 50\%) also require discontinuation of trastuzumab therapy [10]. Both scenarios require cardiac treatment. After normalization of LVEF or resolution of clinical symptoms, the decision to treat with trastuzumab is based on careful risk-benefit analysis for an individual patient. Major risk factors for cardiac side effects are: age over 65 years, prior anthracycline therapy, history of radiotherapy to the left side of the chest, obesity (BMI > $\left.25 \mathrm{~kg} / \mathrm{m}^{2}\right)$, diabetes and hypertension. The study outlines the 
authors' own experience in a risk assessment of cardiac side effects in a group of trastuzumab-treated breast cancer patients.

\section{Material and methods}

The retrospective study was conducted on a group of 120 women treated in the Clinical and Experimental Oncology Department of the Oncology Centre - Maria SklodowskaCurie Memorial Institute (Gliwice Branch), between the years 2006 and 2011. The study group comprised breast cancer patients with HER2 receptor overexpression or HER2 gene amplification. Human epidermal growth factor receptor 2 overexpression was assessed using an immunohistochemical method (IHM) in postoperative specimens or in samples obtained by thick needle biopsy. HER2 gene amplification was additionally assessed by FISH in 8 patients. Inclusion criteria were: baseline LVEF > 50\% and medical history without major pathologies, such as unstable ischaemic heart disease, valvular heart disease, chronic hypertension with cardiovascular problems or uncontrolled diabetes (uncontrolled diabetes was classified as a random blood glucose level $\geq 11.0 \mathrm{mmol} / \mathrm{l}$ in diabetic patients). Patients' characteristics are shown in Table 1.

Echocardiography was performed every 3 months during trastuzumab therapy and also before and after the anthracyclines. Cardiac side effects were assessed according to the NYHA (New York Heart Association) classification and on the CTCAE scale (ver. 4.0). Left ventricular ejection fraction decrease, abnormalities of right ventricular contractility, ventricular dilation and abnormalities of left ventricular contractility were the earliest manifestations of myocardial damage diagnosed by echocardiography.

The following factors were analyzed to determine their potential impact on the development of cardiac side effects: age at disease onset, menopausal status, smoking, hormone receptor status, previous chemotherapy containing anthracyclines, previous radiotherapy to the left side of the chest, comorbidities (diabetes, hypertension, obesity) and baseline LVEF.

Statistical analysis was performed using STATISTICA 7 (StatSoft). Univariate Cox analysis was conducted to assess the impact of risk factors on the development of cardiac side effects. Statistical inference was based on the $p$ value determined using a one-sided test and 95\% confidence intervals. Differences were considered as statistically significant if the $p$ value was $<0.05$. Confounding and effect-modifying variables were studied by multivariate analysis.

\section{Results}

The median age was $54 \pm 10.4$ years (range: $24-71$ years). Twenty percent of patients were older than 60 years. Surgery was performed on 100 (83\%) patients (of whom 20 patients underwent BCT). In 20 (17\%) patients operation was impossible due to advanced stage of disease. The studied group contained 74 (62\%) early breast cancer patients and 46 (38\%) metastatic patients. Adjuvant chemotherapy was given to 113 (94\%) patients and adjuvant radiotherapy to 91 (76\%) patients. FAC (5-fluorouracil $500 \mathrm{mg} / \mathrm{m}^{2}$, adriamycin $50 \mathrm{mg} / \mathrm{m}^{2}$, cyclophosphamide $500 \mathrm{mg} / \mathrm{m}^{2}$ ) was the most com- mon chemotherapy regimen. The other used regimens were AC (adriamycin $60 \mathrm{mg} / \mathrm{m}^{2}$, cyclophosphamide $600 \mathrm{mg} / \mathrm{m}^{2}$ ), AT (adriamycin $50 \mathrm{mg} / \mathrm{m}^{2}$, docetaxel $75 \mathrm{mg} / \mathrm{m}^{2}$ ) and TAC (docetaxel $75 \mathrm{mg} / \mathrm{m}^{2}$, adriamycin $50 \mathrm{mg} / \mathrm{m}^{2}$, cyclophosphamide $500 \mathrm{mg} / \mathrm{m}^{2}$ ). Estrogen receptor expression was identified in 66 (55\%) patients and progesterone receptor expression in $55(45 \%)$ patients. Both steroid receptors were negative in 48 (40\%) women. Trastuzumab was used for adjuvant therapy in 74 (62\%) patients, and for the treatment of metastatic disease in 46 patients (38\%), including 15 patients who underwent immunotherapy in second-line systemic treatment. Adjuvant trastuzumab was given after anthracyclines to 74 (62\%) patients and simultaneously with taxanes to 46 (38\%) patients. Adjuvant therapy consisted of 18 infusions of trastuzumab. Patients treated for metastatic disease received between 2 and 118 infusions (median number: 23).

During trastuzumab therapy, a total of 56 patients (47\%) had reduced LVEF in comparison with baseline values. The LVEF reduction observed in the majority of patients (46 individuals, 39\%) was clinically insignificant, not

Table 1. Patient characteristics

\begin{tabular}{|c|c|c|}
\hline & No. of patients & $\%$ \\
\hline \multicolumn{3}{|l|}{ Age, years } \\
\hline$<65$ years & 108 & 90 \\
\hline$>65$ years & 12 & 10 \\
\hline \multicolumn{3}{|l|}{ Menopausal status } \\
\hline pre-menopause & 31 & 26 \\
\hline post-menopause & 89 & 74 \\
\hline Early breast cancer & 74 & 62 \\
\hline Metastatic breast cancer & 46 & 38 \\
\hline \multicolumn{3}{|l|}{ History of smoking } \\
\hline yes & 37 & 31 \\
\hline no & 83 & 69 \\
\hline \multicolumn{3}{|l|}{ Hormonal receptor status } \\
\hline positive & 48 & 40 \\
\hline negative & 72 & 60 \\
\hline \multicolumn{3}{|l|}{ ER } \\
\hline $\mathrm{ER}+$ & 66 & 55 \\
\hline ER- & 54 & 45 \\
\hline \multicolumn{3}{|l|}{ PR } \\
\hline $\mathrm{PR}+$ & 55 & 45 \\
\hline PR- & 65 & 54 \\
\hline \multicolumn{3}{|l|}{ Radiotherapy } \\
\hline yes & 91 & 76 \\
\hline no & 29 & 24 \\
\hline Priori left-sided radiotherapy & 49 & 54 \\
\hline \multicolumn{3}{|l|}{ Hormone therapy } \\
\hline yes & 71 & 60 \\
\hline no & 48 & 40 \\
\hline \multicolumn{3}{|l|}{ Anthracycline exposure } \\
\hline yes & 109 & 91 \\
\hline no & 11 & 9 \\
\hline cumulative dose $>300 \mathrm{mg} / \mathrm{m}^{2}$ & 10 & 8 \\
\hline Diabetes mellitus & 4 & 3.4 \\
\hline Hypertension & 11 & 9 \\
\hline BMI > 25 (obesity) & 22 & 18 \\
\hline
\end{tabular}


exceeding $10 \%$ of the baseline value. In ten women (8\%), the LVEF reduction exceeded $10 \%$ of the baseline. In five patients the LVEF decrease did not exceed $15 \%$ and in the remaining five it was higher than $15 \%$ of the baseline value. Two patients had an accumulation of fluid in the pericardium. Decompensated heart failure manifesting as a considerable limitation of physical activity, heart palpitations and dyspnea developed in two patients, while abnormal contractility (generalized hypokinesia) occurred in five patients. Among patients requiring cardiac treatment only one woman was older than 65 years. Four patients received trastuzumab for metastatic disease ( $9 \%$ of all patients with metastatic diseases) and six patients in adjuvant treatment (8\% of all patients receiving adjuvant treatment). 109 (91\%) women were previously treated with chemotherapy containing anthracyclines and 49 (54\%) of them received radiotherapy to the left side of the chest. Furthermore, no steroid receptor expression (either ER or PR) in tumor was detected in any of those patients. Appropriate cardiac treatment improved the cardiac status and normalized LVEF in five patients and it allowed immunotherapy to be reintroduced. No cardiac status improvement was achieved in the remaining five patients who required premature discontinuation of immunotherapy. Univariate Cox analysis demonstrated cardiac side effects in $4.2 \%$ of patients previously treated with radiotherapy applied to the left side of the chest, and in $3.3 \%$ of patients who did not have radiotherapy to that area of the body $(p=0.05)$. In the multivariate analysis patients receiving radiotherapy were more likely to develop cardiotoxicity if they were older $(p=0.0003)$. In the analyzed group patients had baseline LVEF ranging between $50 \%$ and $70 \%$. Left ventricular ejection fraction values between $50 \%$ and $60 \%$ were noted in 77 patients $(64 \%)$ and between $60 \%$ and $70 \%$ in 43 women (36\%). Cardiac side effects occurred in nine patients with baseline LVEF between $50 \%$ and $60 \%$, and in one patient whose LVEF was $70 \%(p<0.001)$. No relationship was confirmed between the number of trastuzumab infusions and LVEF decrease. A decrease of LVEF was frequently observed in patients with higher BMI ( $p=0.05)$. A full description of LVEF changes during trastuzumab therapy is included in Table 2.
Cardiac side effects, including symptomatic heart failure, developed in four patients who were previously treated with chemotherapy containing anthracyclines at a dose exceeding $300 \mathrm{mg} / \mathrm{m}^{2}$ and in six patients who were treated with anthracyclines at doses lower than $300 \mathrm{mg} / \mathrm{m}^{2}(p=0.084)$. Negative receptor status (either ER or PR) was detected in ten patients (8\%) with symptoms of cardiac side effects. Evidence of cardiac side effects was noted in $25 \%$ of patients with negative ER or PR tumors in comparison with 72 (60\%) patients with positive receptor status and without cardiotoxicity symptoms $(p=0.045)$. Univariate analysis showed a correlation between cardiac side effects and negative steroid receptor status (ER/PR) compared to positive receptor status ( $6 \%$ vs. $3 \%$ ) ( $p=0.00005)$. In the multivariate analysis ER/PR negative, HER 2 overexpressing breast cancer patients were significantly more prone to develop asymptomatic or symptomatic cardiac events during treatment with trastuzumab if presenting with baseline LVEF between $50 \%$ and $60 \%(p=0.0001)$. No relationship was identified in the study group between cardiotoxicity risk in trastuzumab treatment and the presence of such risk factors as age ( $p=0.465)$, menopausal status ( $p=0.728)$, obesity $(p=0.977)$, hypertension $(p=0.685)$, diabetes $(p=0.537)$ and use of stimulants ( $p=0.891)$.

Sixty-two percent of women in the study group were given trastuzumab in adjuvant treatment and 38\% for metastatic disease of breast cancer. In the group of patients receiving adjuvant therapy, cardiotoxicity occurred in $6.6 \%$ of patients and in patients treated for metastatic disease in $8.7 \%$ of cases, $p=0.710$. Among patients receiving adjuvant treatment a decrease in LVEF was noted in three patients in the initial stage of therapy (4-8 infusions) and in two patients at the end of therapy. As for treatment implemented in metastatic disease, a decrease in LVEF was mainly observed after 20 infusions. In the group treated for metastatic disease there was an insignificantly higher incidence of contractility abnormalities compared with the group receiving adjuvant treatment ( $8.7 \%$ vs. $1.4 \%, p=0.141$ ). Also, there was not significantly higher incidence of fluid accumulation in the pericardial cavity $(2.2 \%$ vs. $1.4 \%, p=0.739)$. There were no differences between the groups in terms of LVEF reduction

Table 2. Symptomatic and asymptomatic cardiac events

\begin{tabular}{|c|c|c|c|c|c|c|}
\hline Cardiotoxicity & NYHA criteria & $\begin{array}{l}\text { No. of risk } \\
\text { factors }\end{array}$ & $\begin{array}{l}\text { LVEF before } \\
\text { trastuzumab (\%) }\end{array}$ & $\begin{array}{l}\text { Lowest LVEF } \\
\text { value (\%) }\end{array}$ & $\begin{array}{l}\text { Trastuzumab } \\
\text { continuation }\end{array}$ & $\begin{array}{c}\text { LVEF after } \\
\text { completion of therapy (\%) }\end{array}$ \\
\hline asymptomatic & $\|$ & 2 & 52 & 45 & yes & 55 \\
\hline symptomatic & III & 3 & 59 & 39 & no & 41 \\
\hline asymptomatic & $\|$ & 1 & 55 & 43 & yes & 53 \\
\hline symptomatic & IV & 1 & 58 & 35 & no & 38 \\
\hline asymptomatic & II & 2 & 60 & 40 & no & 45 \\
\hline asymptomatic & II & 3 & 50 & 35 & no & 40 \\
\hline asymptomatic & I & 2 & 56 & 46 & yes & 51 \\
\hline asymptomatic & 1 & 0 & 58 & 45 & no & 48 \\
\hline asymptomatic & I & 1 & 70 & 55 & yes & 60 \\
\hline asymptomatic & I & 1 & 55 & 45 & yes & 56 \\
\hline
\end{tabular}


Table 3. A comparison of adjuvant therapy with treatment of metastatic disease

\begin{tabular}{|c|c|c|c|c|c|}
\hline \multirow[t]{2}{*}{ Parameter } & \multicolumn{2}{|c|}{$\begin{array}{l}\text { Trastuzumab in } \\
\text { adjuvant treatment }(n=74)\end{array}$} & \multicolumn{2}{|c|}{$\begin{array}{c}\text { Trastuzumab in } \\
\text { metastatic disease }(n=46)\end{array}$} & \multirow[t]{2}{*}{$p$} \\
\hline & $N$ & $\%$ & $N$ & $\%$ & \\
\hline age $>60$ years & 13 & 17.6 & 8 & 17.4 & 0.941 \\
\hline hormonal receptor status (ER/PR-) & 26 & 35.1 & 22 & 47.8 & 0.167 \\
\hline radiotherapy & 57 & 77.0 & 34 & 73.9 & 0.698 \\
\hline hormone therapy & 48 & 64.9 & 23 & 50.0 & 0.088 \\
\hline anthracycline exposure & 66 & 89.2 & 43 & 93.5 & 0.428 \\
\hline cardiotoxicity & 5 & 6.6 & 4 & 8.7 & 0.710 \\
\hline decrease in LVEF & 6 & 8.1 & 4 & 8.7 & 0.852 \\
\hline cardiac arrhythmias & 1 & 1.4 & 4 & 8.7 & 0.141 \\
\hline changes of pericarditis & 1 & 1.4 & 1 & 2.2 & 0.739 \\
\hline adverse events & 2 & 2.7 & 1 & 2.2 & 0.656 \\
\hline
\end{tabular}

( $8.7 \%$ vs. $8.1 \%)$ or symptomatic heart failure $(2.2 \%$ vs. $2.1 \%)$ (Table 3).

\section{Discussion}

Trastuzumab is the standard care for patients with HER2 (+) breast cancer both for adjuvant therapy and treatment of metastatic disease. Trastuzumab can be combined with chemotherapy regimens containing anthracyclines and taxanes or used in monotherapy. Four major studies of trastuzumab in adjuvant treatment (HERA, NSABP B31, Fin$\mathrm{HER}$, and BCIRG 006) showed that the addition of trastuzumab to adjuvant therapy significantly extended disease-free survival (DFS) and overall survival (OS) without a significant effect on toxicity with the exception of cardiotoxicity in trastuzumab-treated patients [11-16]. The HERA trial demonstrated that trastuzumab in adjuvant treatment significantly increased 4-year OS rates (89\% vs. 87\%) and 4-year DFS rates (78\% vs. $72 \%$ ) in comparison with trastuzumab-free therapy [11]. The NSABP B31 and N9831 trials demonstrated that addition of trastuzumab to chemotherapy regimen (paclitaxel following $\mathrm{AC}$ ) reduces disease recurrence by $52 \%$ and risk of death by $33 \%$ compared with chemotherapy alone $[16,17]$. In the PACSO4 study, which was a randomized phase III trial of women with node positive early breast cancer, the results contrast with the above-mentioned trials. The results of this study showed that patients who received trastuzumab had a non-significant, 14\% reduction in the risk of relapse [18].

Some of the patients experiencing cardiac side effects exhibit typical symptoms of heart failure including reduced exercise tolerance, dyspnea on exertion, tachycardia or signs of fluid retention. But the most common cardiotoxicity seen with trastuzumab is asymptomatic decline in LVEF. In most patients cardiac dysfunction was reversible on discontinuation of trastuzumab therapy and introduction of cardiac therapy $[2,13]$. In the HERA trial asymptomatic decline in LVEF was observed in $7.1 \%$ of patients and symptoms of heart failure were seen in $1.7 \%$ of trastuzumab treated patients [11]. Similarly, in the NSABP B31 and N9831 trials $14.2 \%$ of patients discontinued trastuzumab therapy because of asymptomatic decline in LVEF and only $4.7 \%$ of patients discontinued therapy due to heart failure $[16,17]$. In a study by Serrano et al., performed in elderly patients (age > 70) who were given trastuzumab both in adjuvant therapy and for treatment of metastatic disease, cardiac side effects triggered by trastuzumab developed in $26.7 \%$ of patients, including symptoms of heart failure in $8.9 \%$ [19]. In the study group an asymptomatic decrease in LVEF was noted in $37 \%$ of patients. Cardiotoxicity occurred in $8 \%$ of women, including $2 \%$ of patients with heart failure symptoms. Cardiac side effects developed in nine patients with baseline LVEF between $50 \%$ and $60 \%$, and in just one patient whose baseline LVEF was $70 \%$ ( $p$ <0.001). The increase of LVEF was frequently observed in patients with higher BMI ( $p=0.05)$.

Patient age and prior anthracycline therapy are recognized independent risk factors for trastuzumab-induced cardiotoxicity $[4,20]$. Age $>60$ years was found to be a risk factor in the NSABP B31 trial [16]. An association between increasing age and cardiac toxicity was also observed in the NCTG N9831 trial, in which $15 \%$ of patients were aged $\geq 60$. In contrast, in the BCIRG 006 trial an influence of older age on cardiac side effects was not reported [13]. In this studied group, older age and history of cardiac diseases had no significant impact on the development of cardiotoxicity. Only patients receiving radiotherapy were more likely to develop cardiotoxicity if they were older $(p=0.0003)$. Trastuzumab combination with anthracycline-containing chemotherapy regimens increased the incidence of cardiac side effects even to $27 \%$ [3]. The risk of cardiac dysfunction increases after a cumulative dose of doxorubicin exceeding $300 \mathrm{mg} / \mathrm{m}^{2}$ [20]. The addition of trastuzumab to anthracycline-containing chemotherapy was associated with a higher incidence of heart failure (NYHA III-IV) compared with the combination of trastuzumab with anthracycline-free chemotherapy (BCIRG trial) [13]. In this study $90 \%$ of patients received anthracycline-containing therapy, including $23 \%$ who received anthracycline doses exceeding $300 \mathrm{mg} / \mathrm{m}^{2}$. The HERCULES multicenter, phase I to II trial was conducted to evaluate the cardiac safety of epirubicin/cyclophosphamide plus trastuzumab reg- 
imen as first line therapy for HER2-positive metastatic breast cancer patients. In this regimen the incidence of doselimiting cardiotoxicity was lower in comparison with trastuzumab and doxorubicin [22]. Prior anthracycline treatment was not an independent risk factor for trastuzumabinduced cardiotoxicity in the current study. The small size of the sample may have impacted these results.

Other factors elevating the risk of cardiac side effects are: obesity, hypertension, diabetes and previous radiotherapy to the left side of the chest $[3,21]$. In the HERA trial, lower baseline LVEF (55-60\%), high BMI (> 25), hyperlipidemia and higher cumulative dose of anthracycline (over $287 \mathrm{mg} / \mathrm{m}^{2}$ ) proved to be significant risk factors for cardiotoxicity. On the other hand, diabetes, history of cardiac diseases or hypertension had no significant effect on the development of cardiotoxicity [11, 20]. In a study by Serrano et al., history of cardiac diseases and diabetes also had a major effect on the development of cardiotoxicity in the elderly patient population. Hypertension, trastuzumab dose, history of radiotherapy to the left side of the chest and use of stimulants were not found to have any effect on cardiac side effects [19]. The discrepancies in study results may arise from differences in endpoints, inclusion criteria and therapeutic regimens. Statistically significant risk factors for cardiac toxicity in the analyzed group of patients included: lower (50-60\%) baseline LVEF ( $p<0.001)$, high BMI (> 25), negative steroid receptor status (ER/PR) ( $p=0.045)$ and history of radiotherapy applied to the left side of the chest $(p=0.05)$. ER/PR negative, HER2 overexpressing breast cancer patients were significantly more likely to develop cardiac events during treatment with trastuzumab if presenting with baseline LVEF between $50 \%$ and $60 \%(p=0.0001)$. The myocardium is a target tissue for estrogen, which plays an important role in the pathogenesis of cardiovascular disease. Myocytes contain functional receptors (ERs) ER $\alpha$ and ER 3 . A study with ER knockout animals showed that ERs are required for cardioprotection in myocardial ischemia and pressure overload left ventricular hypertrophy [23]. The influence of negative steroid receptor status on cardiac toxicity may be connected with the lack of cardioprotective effects of estrogens.

A comparison of adjuvant therapy with treatment for metastatic disease revealed no statistically significant difference between two patient groups in terms of cardiac side effects including contractility disorders, pericardial abnormalities (fluid accumulation in the pericardium), LVEF decrease or symptomatic heart failure. Differences in the number of patients in both groups may also be responsible for the lack of significant correlation between cardiotoxicity and anthracycline-containing chemotherapy ( $p=0.08)$. Baseline LVEF > 55\%, longer interval between anthracycline-containing chemotherapy, radiotherapy and immunotherapy, and more frequent cardiac assessment by echocardiography are associated with lower cardiotoxicity of treatment [20, 24].

\section{Summary}

In the analyzed group of patients, risk factors for cardiac toxicity included: previous radiotherapy to the left side of the chest $(p=0.05)$, high BMI, low baseline LVEF $(p<0.001)$ and negative steroid receptor status $(p=0.045)$. The impact of anthracycline-containing chemotherapy was not significant $(p=0.08)$. Patients receiving radiotherapy were more prone to develop cardiotoxicity if they were older $(p=0.0003)$. Factors such as diabetes, hypertension, menopausal status, use of stimulants or age of the patients did not affect the development of cardiotoxicity. Moreover, cancer advancement was not found to have any prognostic value for cardiac side effects. There was no significant difference between patients receiving adjuvant therapy and patients treated for metastatic disease.

The authors declare no conflict of interest.

\section{References}

1. Slamon DJ, Clark GM, Wong SG, Levin WJ, Ullrich A, McGuire WL Human breast cancer: correlation of relapse and survival with amplification of the HER-2/neu oncogene. Science 1987; 235: 177-82.

2. Yeh ET, Tong AT, Lenihan DJ, et al. Cardiovascular complications of cancer therapy: diagnosis, pathogenesis, and management. Circulation 2004; 109: 3122-31.

3. Seidman A, Hudis C, Pierri MK, et al. Cardiac dysfunction in the trastuzumab clinical trials experience. J Clin Oncol 2002; 20: 1215-21.

4. Keefe DL. Trastuzumab - associated cardiotoxicity. Cancer 2002; 95: 1592-600.

5. Freedman NJ, Ginsburg GS. Novel - and "neu" - therapeutic possibilities for heart failure. J Am Coll Cardiol 2006; 48: 1448-50.

6. de Korte MA, de Vries EG, Lub-de Hooge MN, et al. 111Indium trastuzumab visualises myocardial human epidermal growth factor receptor 2 expression shortly after anthracycline treatment but not during heart failure: a clue to uncover the mechanisms of trastuzumab - related cardiotoxicity. Eur J Cancer 2007; 43: 2046-51.

7. Sengupta PP, Northfelt DW, Gentile F, Zamorano JL, Khandheria BK. Trastuzumab-induced cardiotoxicity: heart failure at the crossroads. Mayo Clin Proc 2008; 83: 197-203.

8. Fox KF. The evaluation of left ventricular fraction for patients being considered for, or receiving Trastuzumab (Herceptin) therapy [letter]. Br J Cancer 2006; 95: 1454.

9. Stoodley PW, Richards DA, Meikle SR, Clarke J, Hui R, Thomas L. The potential role of echocardiographic strain imaging for evaluating cardiotoxicity due to cancer therapy. Heart Lung Circ 2011; 20: 3-9.

10. Duchnowska R, Szmidt S, Szczylik C, Opolski G. Trudności w monitorowaniu echokardiograficznym leczenia trastuzumabem chorych na raka piersi - opis przypadku i przegląd zaleceń. Kardiol Pol 2008; 66: 895-8.

11. Piccart-Gebhart MJ, Procter M, Leyland-Jones B, et al.; Herceptin Adjuvant (HERA) Trial Study Team. Trastuzumab after adjuvant chemotherapy in HER2 - positive breast cancer. N Engl J Med 2005; 353: 1659-72.

12. Romond EH, Perez EA, Bryant J, et al. Trastuzumab plus adjuvant chemotherapy for operable HER2 - positive breast cancer. N Engl J Med 2005; 353: 1673-84.

13. Slamon D, Eiermann W, Robert N, et al. Phase III randomized tria comparing doxorubicin and cyclophosphamide followed by docetaxel (ACT) with doxorubicin and cyclophosphamide followed by docetaxel and trastuzumab (A?T) with docetaxel, carboplatin, and trastuzumab (THC) in HER2 positive early breast cancer patients: BCIRG 006 study [abstract]. Breast Cancer Res Treat 2006; 100 (Suppl 1): 52.

14. Joensuu H, Kellokumpu-Lehtinen PL, Bono P, et al. Adjuvant docetaxel or vinorelbine with or without trastuzumab for breast cancer. N Engl J Med 2006; 354: 809-20.

15. Costa RB, Kurra G, Greenberg L, Geyer CE. Efficacy and cardiac safety of adjuvant trastuzumab-based chemotherapy regimens for HER2positive early breast cancer. Ann Oncol 2010; 21: 2153-60. 
16. Tan-Chiu E, Yothers G, Romond E, et al. Assessment of cardiac dysfunction in a randomized trial comparing doxorubicin and cyclophosphamide followed by paclitaxel, with or without trastuzumab as adjuvant therapy in node positive, human epidermal growth factor receptor 2 overexpressing breast cancer: NSABP B-31. J Clin Oncol 2005; 23: 7811-9.

17. Perez EA, Suman VJ, Davidson NE, et al. Cardiac safety analysis of doxorubicin and cyclophosphamide followed by paclitaxel with or without trastuzumab in the North Central Cancer Treatment Group N9831 Adjuvant Breast Cancer Trial. J Clin Oncol 2008; 26: 1231-8.

18. Spielmann M, Roché H, Delozier T, et al. Trastuzumab for patients with axillary-node-positive breast cancer: Results of the FNCLCC-PACS 04 trial. J Clin Oncol 2009; 27: 6129-34.

19. Serrano C, Cortés J, De Mattos-Arruda L, et al. Trastuzumab - related cardiotoxicity in the elderly: a role for cardiovascular risk factors. Ann Oncol 2012; 23: 897-902.

20. Suter TM, Procter M, van Veldhuisen DJ, et al. Trastuzumab - associated cardiac adverse effects in the herceptin adjuvant trial. J Clin Oncol 2007; 25: 3859-65.

21. Perez EA, Rodeheffer R. Clinical cardiac tolerability of trastuzumab. J Clin Oncol 2004; 22: 322-9.

22. Untch M, Muscholl M, Tjulandin S, et al. First-line trastuzumab plus epirubicin and cyclophosphamide therapy in patients with human epidermal growth factor receptor 2-positive metastatic breast cancer: cardiac safety and efficacy data from the Herceptin, Cyclophosphamide, and Epirubicin (HERCULES) trial. J Clin Oncol 2010; 28: 1473-80.

23. Deroo BJ, Korach KS. Estrogen receptors and human diseases. J Clin Invest 2006; 116: 561-8.

24. Untch M, Rrezai M, Loibl S, et al. Neoadjuvant treatment of HER2 overexpression primary breast cancer with Trastuzumab given concomitantly to epirubicin/cyclophosphamide followed by docetaxe \pm capecitabine. First analysis of efficacy and safety of the GBG/AGO multicancer Intergroup - study “GeparQuattro”. Eur J Cancer 2008; 6: 47.

\section{Address for correspondence}

\section{Dr Joanna Huszno}

Clinical and Experimental Oncology Department

Maria Skłodowska-Curie Memorial Cancer Center and Institute of Oncology

Gliwice Branch

Wybrzeże Armii Krajowej 15

44-101 Gliwice, Poland

e-mail: joahus@wp.pl

Submitted: 23.07 .2012

Accepted: 22.10 .2012 